\title{
ACOUSTIC INFLUENCE ON THE VIBRATION OF A CYLINDRICAL MEMBRANE DRUM FILLED WITH A COMPRESSIBLE FLUID
}

\author{
MANUEL GASCÓN-PÉREZ \\ Escuela Técnica Superior de Ingeniería Aeronáutica y del Espacio, UPM, \\ 28040 Madrid, Spain \\ manuel.gascon@upm.es
}

Received

Revised

\begin{abstract}
The study of the dynamic behaviour of a membrane in contact with a fluid is interesting due to the numerous applications in technology. The vibro-acoust ic behaviour of a circular membrane in a cylindrical container or a membrane drum filled with a non-viscous fluid is analyzed. A boundary element method is used and the acoustic pressure over the boundary is calculated employing the Kirchhoff`s integral equation and that with the equation of motion of the membrane, the natural frequencies of vibration are obtained. Furthermore the effect of the drum height, drum radius, membrane material density, tension parameter and fluid density on the frequencies are evaluated, as well as the variation of the fluid mass coefficient with the wave number. Validation of the method is made comparing the results with those obtained by other authors and theories.
\end{abstract}

Keywords: membrane drum; fluid-structure interaction; natural frequencies; boundary element method.

\section{Introduction}

Investigations and studies of vibrating membranes have been conducted for many years. These structures formed from thin-walled material can be found in various applications from biological to architectural and aerospace, in which the tangential and bending reaction to loads are negligible. Motivations for such studies were the solution of practical problems, for example the investigation of acoustics of musical instruments such as drums and bells, architectural or civil structures, diaphragms in transducers, biomedical prosthesis such as artificial organs, and space based applications such as radio antennas and optical reflectors, see Jenkins et al. [2006]. All of them can be considered as different types of fluid-structure interaction problems, as an example the recent works of Soltani and Reddy [2015] who analyze the vibration of elastic plates in contact with an inviscid fluid, Abassi et al. [2016] who analyze the torsional vibration of a fluidfilled multilayered transversely is otropic finite circular cylinder, Erchiqui et al. [2015] who carry out an study of characterization of polymeric membranes under large deformations using fluidstructure coupling, and lastly Zhao and $\mathrm{Li}$ [2015] who undertake the study of vibration and acoustic responses of an orthotropic composite conical shell in a hygroscopic environment. The interactions between fluid and membranes affect the frequency characteristics of the coupled system and could change the performance of the system under dynamic loading. For these light structures, even when the fluid is air, the contribution to the dynamic properties can be important.

The problem of fluid-membrane interactions has been investigated by many researchers. Bauer [1995] considered the coupled frequencies of a liquid in a circular cylindrical container with an 
elastic liquid surface cover; for this an analytic method is employed considering the deformation equation of the membrane in which the pressure force is obtained through the velocity potential using Bernouilli's linearized equation. Chiba et al .[2002] considered the free hydroelastic vibration in a cylindrical container with a membrane bottom, containing liquid with surface tension. Shabani et al. [2013] studied the hydroelastic vibration of a circular diaphragm in the fluid chamber of a reciprocating micro pump. Tariverdilo et al. [2012, 2013] considered the free vibration of a circular plate or membrane situated on the bottom of a cylindrical container filled with an incompressible and non-viscous fluid, for which it applies a variational approach taking into account the deformation of the plate and the velocity potential function for the fluid including also its boundary conditions. Kyeong et al. [2005] analyzed the hydro-elastic vibration of a circular plate clamped at an off center location of a rigid cylindrical container with the fluid bisected by the plate in the container; for this the fluid was considered non-viscous and compressible and an analytical method was developed by the Rayleigh-Ritz method based on the Fourier-Bessel series expansion. Xiang et al. [2016] used a variational formulation for vibroacoustic analysis of a panel backed by an irregularly-bounded cavity.

The vibration of a circular membrane clamped on the bottom of a cylindrical container filled with fluid (compressible or incompressible) is analyzed in this paper. Applying a boundary element method taking into account the Kirchhoff`s integral formulation for the fluid pressure and the equation of motion of the membrane, the frequencies of the coupled systemare evaluated by a collocation method leading to an eigenvalue problem. The advantage of the boundary element method with respect to the finite element method is that it requires a discretization of only the boundary of the fluid domain and not the entire fluid domain, so that for example in the case of an infinite domain the application of the finite element method has limitations. This method is characterized by its relative simplicity, nevertheless it requires the calculation of singular integrals that are solved by taking their principal value.

Finally the effects of fluid parameters such as depth and density, and the membrane parameters such as tension, material density and thickness of the free vibration response on the coupled system are investigated as well as the variation of the fluid mass coefficient with the wave number.

\section{Problem Formulation}

The equation of the membrane deformation in contact with the fluid the fluid is, see Wang [2014]:

$$
T \Delta w(r, \theta, t)-\rho_{m} t_{h} \frac{\partial^{2} w}{\partial t^{2}}=p(r, \theta, t)
$$

with $w$ the membrane deformation, $T$ the membrane tension, $t_{h}$ the membrane thickness, and $\rho_{m}$ the material density of the membrane.

It is supposed that the only action over the membrane is the fluid pressure $p$ inside the drum in contact with it.

This pressure distribution can be obtained by solving the wave equation on the fluid domain for a still fluid

with $a_{\infty}$ the sound speed.

$$
\frac{1}{a_{\infty}^{2}} \frac{\partial^{2} p}{\partial t^{2}}-\Delta p=0
$$

Application of the momentum equation at the surface of the membrane that is placed at the $z=0$ plane and with a radius, yields the boundary condition:

$$
\frac{\partial p}{\partial z}=-\rho_{\infty} \frac{\partial^{2} w}{\partial t^{2}} \text { at } z= \pm 0 \quad \text { and } 0<r<a
$$


with $a$ being the radius of the membrane and $\rho_{\infty}$ the fluid density.

And for the rigid walls $\frac{\partial p}{\partial n}=0$, with $\vec{n}$ the normal to the surface of the wall.

In Fig.1 the geometrical characteristics are presented which define the membrane drum.

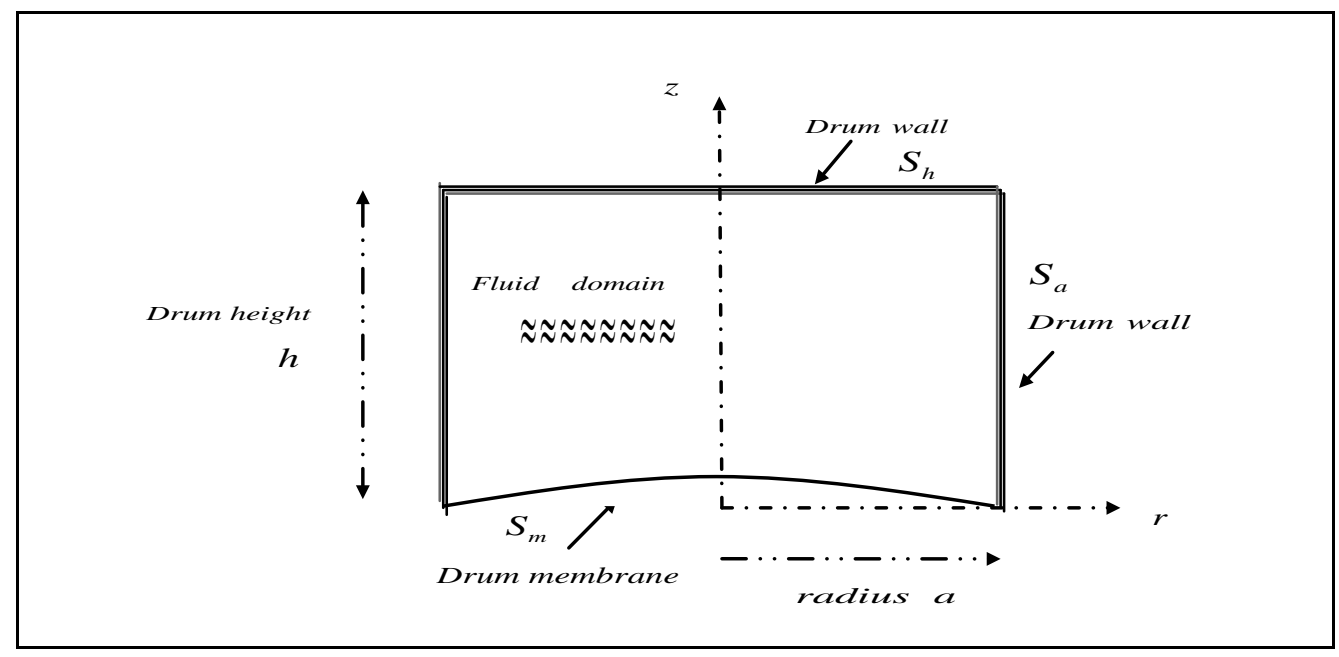

Fig. 1. Scheme of a membrane drum showing the different geometrical parameters

If transient motion has faded out, the motion of both the fluid and the structure would be assumed to be harmonic

$$
w(r, \theta, t)=\tilde{w}(r, \theta) \cdot e^{-i \omega t} \text { and } p(r, \theta, z, t)=\tilde{p}(r, \theta, z) \cdot e^{-i \omega t}
$$

The deformation of the membrane, when coupled with the fluid, will be expressed as a linear combination of the normal modes of the membrane in vacuum

$$
\tilde{w}(r, \theta)=\sum_{m} \sum_{n} W_{m}^{n}(r) \cdot \cos (m \theta) q_{m}^{n}
$$

where $q_{m}^{n}$ represents the weight coefficients that indicate the contribution of the mode $W_{m}^{n}$ to the deformation of the membrane.

The functions $W_{m}^{n}(r)$ have the general expression, see Wang [2014]:

$$
W_{m}^{n}(r)=J_{m}\left(\beta_{m}^{n} r\right)
$$

where $J_{m}$ is the Bessel function of $m^{\text {th }}$ order, and $\beta_{m}^{n}$ is a parameter that depends on the dynamic characteristics of the membrane and provides the frequencies of the membrane in vacuum. The indexes $m, n$ represent the number of nodal diameters and nodal circles respectively.

Once the assumption of harmonic motion is made, equation (2.2) is expressed as

$$
\Delta \tilde{p}+k^{2} \tilde{p}=0
$$

where $k=\frac{\omega}{a_{\infty}}$ is the wave number, with $a_{\infty}$ the sound speed.

If at the boundary condition, equation (2.3), equations (2.4) and (2.5) are substituted, it is obtained

$$
\left.\frac{\partial \tilde{p}(r, \theta, z)}{\partial z}\right|_{z=0}=\rho_{\infty} \omega^{2} \tilde{w}(r, \theta)=\rho_{\infty} \omega^{2} \sum_{n} \sum_{m} W_{m}^{n}(r) \cdot \cos (m \theta) q_{m}^{n}
$$

at $\quad Z=0$ and $0<r<a$ 
Then the pressure field can also be expanded in harmonic functions of $\theta$

$$
\tilde{p}(r, \theta, z)=\sum_{m} \sum_{n} P_{m}^{n}(r, z) \cdot \cos (m \theta)
$$

And now equation (2.8) can be expressed as

$$
\left.\frac{\partial P_{m}^{n}}{\partial z}\right|_{z=0}=\rho_{\infty} \omega^{2} W_{m}^{n}(r) q_{m}^{n} \quad 0 \leq r \leq a
$$

By substitution of equation (2.9) at (2.7) the differential equation for the pressure mode $P_{m}^{n}$ is

$$
\frac{\partial^{2} P_{m}^{n}}{\partial z^{2}}+\frac{\partial^{2} P_{m}^{n}}{\partial r^{2}}+\frac{1}{r} \frac{\partial P_{m}^{n}}{\partial r}-\frac{m^{2}}{r^{2}} P_{m}^{n}+k^{2} P_{m}^{n}=0
$$

\section{Calculus of the pressure over the membrane}

Considering the fluid boundary, see Fig.1, and applying the Kirchhoff-Helmholtz's integral equation for the pressure mode (that for simplicity is expressed as $P(r, z)$ ) (from Refs. Crocker, M.J. [2007] and Fahy, F. [2001] )

$$
P(r, z)=\iint_{S}\left(P \frac{\partial g}{\partial n}-g \frac{\partial P}{\partial n}\right) d S
$$

with $g$ the Green`s function for the non-homogeneous Helmholtz's equation that satisfies the Sommerfield's radiation condition, whose solution is

$$
g=-\frac{1}{4 \pi} \frac{e^{i k R}}{R}
$$

where $R$ is the distance between the source point and the field point, $R=\sqrt{(r-\rho)^{2}+(z-\zeta)^{2}}$

The surface boundary $S$ is formed by the membrane surface $S_{m}$, the cylindrical rigid surface of the drum $S_{a}$ and the circular rigid surface of the drum $S_{h}$.

Considering the pressure $P(r, z)$ on the boundary and its normal gradient $\frac{\partial P}{\partial \tilde{n}}$, the following integral equation is obtained:

$$
\frac{\partial P(r, z)}{\partial \tilde{n}}=\iint_{S}\left(P \frac{\partial^{2} g}{\partial n \partial \tilde{n}}-\frac{\partial g}{\partial \tilde{n}} \frac{\partial P}{\partial n}\right) d S
$$

Considering the different surface boundaries and taking into account that $\frac{\partial P}{\partial n}=0$ for the rigid boundaries $S_{a}$ and $S_{h}$

$$
\frac{\partial P(r, z)}{\partial \tilde{n}}=\iint_{S_{h}} P \frac{\partial^{2} g}{\partial n \partial \tilde{n}} d S+\iint_{S_{a}} P \frac{\partial^{2} g}{\partial n \partial \tilde{n}} d S+\iint_{S_{m}}\left(P \frac{\partial^{2} g}{\partial n \partial \tilde{n}}-\frac{\partial g}{\partial \tilde{n}} \frac{\partial P}{\partial n}\right) d S
$$

Considering the three boundaries of the drum and equation (2.15), a system of three integral equations is obtained, and by applying a collocation method and solving this system, the pressure field over these boundaries is calculated, i.e. the pressure over the membrane $P$, the pressure over the cylindrical rigid surface $P_{a}$, and the pressure over the circular rigid surface $P_{h}$

Applying the integral equation of the normal pressure gradient for the cylindrical rigid boundary and taking into account that $\frac{\partial P(a, z)}{\partial r}=0$ 


$$
\left.\left.\left.\left.0=\int_{0}^{h} P(a, \zeta) \frac{\partial^{2} g}{\partial \rho \partial r}\right)_{\substack{r=a \\ \rho=a}} 2 \pi a d \zeta+\int_{0}^{a} P(\rho, h) \frac{\partial^{2} g}{\partial \zeta \partial r}\right)_{\substack{r=a \\ \zeta=h}} 2 \pi \rho d \rho+\int_{0}^{a}\left[-P(\rho, 0) \frac{\partial^{2} g}{\partial \zeta \partial r}\right)_{\substack{r=a \\ \zeta=0}}-\frac{\partial g}{\partial r}\right)_{\substack{r=a \\ \zeta=0}} \rho_{\infty} \ddot{W}\right] 2 \pi \rho d \rho
$$

Identifying $P_{a}(z)=P(a, z), P_{h}(r)=P(r, h)$ and $P(r)=P(r, 0)$ and applying a collocation method, the following system is obtained:

$$
0=\left[I_{a a}\right]\left\{P_{a}\right\}+\left[I_{a h}\right]\left\{P_{h}\right\}+\left[I_{a p}\right]\{P\}+\left[I_{a W}\right]\{\ddot{W}\}
$$

where the influence matrices are expressed:

$$
\left.\left[I_{a a}\right]_{i j}=I_{a a}\left(z_{i}, z_{j}\right)=\int_{\zeta_{j}}^{\zeta_{j+1}} \frac{\partial^{2} g}{\partial \rho \partial r}\right)_{\substack{r=a \\ \rho=a}} 2 \pi a d \zeta=\frac{1}{2} \int_{\zeta_{j}}^{\zeta_{j+1}} \Psi(R) a d \zeta
$$

where $z_{j}=\frac{\zeta_{j}+\zeta_{j+1}}{2}, \quad \Psi=\frac{e^{i k R}}{R^{2}}\left(i k-\frac{1}{R}\right)$ and $R=\sqrt{\left(z_{i}-\zeta\right)^{2}}=\left|z_{i}-\zeta\right|$

Special care must be taken of the singularity when $z_{i}=z_{j}$. To avoid this singularity, the kernel of the integral is split into a regular part and a singular part, see Gascón-Pérez et al [2015]. The regular part is integrated numerically and for the singular part, the principal value, see Roussos [2014], is taken, i.e. $\left[I_{a a}\right]_{i j}=\left[I_{a a}^{r}\right]_{i j}+\left[I_{a a}^{s}\right]_{i j}$

$$
\left.\left[I_{a h}\right]_{i j}=I_{a p}\left(z_{i}, r_{j}\right)=\int_{\rho_{j}}^{\rho_{j+1}} \frac{\partial^{2} g}{\partial \zeta \partial r}\right)_{\substack{r=a \\ \zeta=h}} 2 \pi \rho d \rho=\frac{1}{2} \int_{\rho_{j}}^{\rho_{j+1}} \frac{\Psi_{R}(R)}{R}(a-\rho)\left(z_{i}-h\right) \rho d \rho
$$

where $r_{j}=\frac{\rho_{j}+\rho_{j+1}}{2}, \frac{\partial \Psi}{\partial R}=\Psi_{R}=i k \Psi-\frac{2}{R} \Psi+\frac{e^{i k R}}{R^{4}} \quad$ and $\quad R=\sqrt{(a-\rho)^{2}+\left(z_{i}-h\right)^{2}}$

$$
\left.\left[I_{a p}\right]_{i j}=I_{a p}\left(z_{i}, r_{j}\right)=-\int_{\rho_{j}}^{\rho_{j+1}} \frac{\partial^{2} g}{\partial \zeta \partial r}\right)_{\substack{r=a \\ \zeta=0}} 2 \pi \rho d \rho=-\frac{1}{2} \int_{\rho_{j}}^{\rho_{j+1}} \frac{\Psi_{R}(R)}{R}(a-\rho) z_{i} \rho d \rho
$$

where $R=\sqrt{(a-\rho)^{2}+\left(z_{i}\right)^{2}}$

$$
\left.\left[I_{a W}\right]_{i j}=I_{a W}\left(z_{i}, r_{j}\right)=-\rho_{\infty} \int_{\rho_{j}}^{\rho_{j+1}} \frac{\partial g}{\partial r}\right)_{\substack{r=a \\ \zeta=0}} 2 \pi \rho d \rho=\frac{1}{2} \rho_{\infty} \int_{\rho_{j}}^{\rho_{j+1}} \Psi(R) \cdot(a-\rho) \rho d \rho
$$

where $\quad R=\sqrt{(a-\rho)^{2}+\left(z_{i}\right)^{2}}$

Applying the integral equation of the normal pressure gradient for the circular rigid boundary $S_{h}$ and taking into account that $\frac{\partial P(r, h)}{\partial z}=0$

$$
\left.\left.\left.\left.0=\int_{0}^{h} P(a, \zeta) \frac{\partial^{2} g}{\partial \rho \partial z}\right)_{\substack{\rho=a \\ z=h}} 2 \pi a d \zeta+\int_{0}^{a} P(\rho, h) \frac{\partial^{2} g}{\partial \zeta \partial z}\right)_{\substack{\zeta=h \\ z=h}} 2 \pi \rho d \rho+\int_{0}^{a}\left[-P(\rho, 0) \frac{\partial^{2} g}{\partial \zeta \partial z}\right)_{\substack{\zeta=0 \\ z=h}}-\frac{\partial g}{\partial z}\right)_{\substack{\zeta=0 \\ z=h}} \rho_{\infty} \ddot{W}\right] 2 \pi \rho d \rho
$$

Identifying $P_{a}(z)=P(a, z), P_{h}(r)=P(r, h)$ and $P(r)=P(r, 0)$ and applying a collocation method, the following system is obtained:

where the influence matrices are expressed:

$$
0=\left[I_{h a}\right]\left\{P_{a}\right\}+\left[I_{h h}\right]\left\{P_{h}\right\}+\left[I_{h p}\right]\{P\}+\left[I_{h W}\right]\{\ddot{W}\}
$$

$$
\left.\left[I_{h a}\right]_{i j}=I_{h a}\left(r_{i}, z_{j}\right)=\int_{\zeta_{j}}^{\zeta_{j+1}} \frac{\partial^{2} g}{\partial \rho \partial z}\right)_{\substack{\rho=a \\ z=h}} 2 \pi a d \zeta
$$




$$
\left.\left[I_{h h}\right]_{i j}=I_{h h}\left(r_{i}, r_{j}\right)=\int_{\rho_{j}}^{\rho_{j+1}} \frac{\partial^{2} g}{\partial \zeta \partial z}\right)_{\substack{\zeta=h \\ z=h}} 2 \pi \rho d \rho
$$

Again, special care must be taken of the singularity when $r_{i}=r_{j}$. To avoid this singularity, the kernel of the integral is split into a regular part and a singular part. The regular part is integrated numerically and for the singular part, the principal value is taken, i.e. $\left[I_{h h}\right]_{i j}=\left[I_{h h}^{r}\right]_{i j}+\left[I_{h h}^{s}\right]_{i j}$

$$
\begin{aligned}
& \left.\left[I_{h p}\right]_{i j}=I_{h p}\left(r_{i}, r_{j}\right)=-\int_{\rho_{j}}^{\rho_{j+1}} \frac{\partial^{2} g}{\partial \zeta \partial z}\right)_{\substack{\zeta=0 \\
z=h}} 2 \pi \rho d \rho \\
& \left.\left[I_{h W}\right]_{i j}=I_{h W}\left(r_{i}, r_{j}\right)=-\int_{\rho_{j}}^{\rho_{j+1}} \frac{\partial g}{\partial z}\right)_{\substack{\zeta=0 \\
z=h}} \rho_{\infty} 2 \pi \rho d \rho
\end{aligned}
$$

Applying the integral equation of the normal pressure gradient for the circular membrane and taking into account that $\frac{\partial P(r, 0)}{\partial z}=-\rho_{\infty} \ddot{W}$ and that $\left.\frac{\partial g}{\partial z}\right)_{\substack{z=0 \\ \zeta=0}}=0$

$$
\left.\left.\left.-\rho_{\infty} \ddot{W}=\int_{0}^{h} P(a, \zeta) \frac{\partial^{2} g}{\partial \rho \partial z}\right)_{\substack{\rho=a \\ z=0}} 2 \pi a d \zeta+\int_{0}^{a} P(\rho, h) \frac{\partial^{2} g}{\partial \zeta \partial z}\right)_{\substack{\zeta=h \\ z=0}} 2 \pi \rho d \rho+\int_{0}^{a}\left[-P(\rho, 0) \frac{\partial^{2} g}{\partial \zeta \partial z}\right)_{\substack{\zeta=0 \\ z=0}}\right] 2 \pi \rho d \rho
$$

identifying $P_{a}(z)=P(a, z), \quad P_{h}(r)=P(r, h)$ and $P(r)=P(r, 0)$ and applying a collocation method, the following system is obtained:

$$
0=\left[I_{p a}\right]\left\{P_{a}\right\}+\left[I_{p h}\right]\left\{P_{h}\right\}+\left[I_{p p}\right]\{P\}+\left[I_{p W}\right]\{\ddot{W}\}
$$

where the influence matrices are expressed:

$$
\begin{aligned}
& \left.\left[I_{p a}\right]_{i j}=I_{p a}\left(r_{i}, z_{j}\right)=\int_{\zeta_{j}}^{\zeta_{j+1}} \frac{\partial^{2} g}{\partial \rho \partial z}\right)_{\substack{\rho=a \\
z=0}} 2 \pi a d \zeta \\
& \left.\left[I_{p h}\right]_{i j}=I_{p h}\left(r_{i}, r_{j}\right)=\int_{\rho_{j}}^{\rho_{j+1}} \frac{\partial^{2} g}{\partial \zeta \partial z}\right)_{\substack{\zeta=h \\
z=0}} 2 \pi \rho d \rho \\
& \left.\left[I_{p p}\right]_{i j}=I_{p p}\left(r_{i}, r_{j}\right)=-\int_{\rho_{j}}^{\rho_{j+1}} \frac{\partial^{2} g}{\partial \zeta \partial z}\right)_{\substack{\zeta=0 \\
z=0}} 2 \pi \rho d \rho
\end{aligned}
$$

Again, special care must be taken of the singularity when $r_{i}=r_{j}$. To avoid this singularity, the kernel of the integral is split into a regular part and a singular part. The regular part is integrated numerically and for the singular part, the principal value is taken, i.e. $\left[I_{p p}\right]_{i j}=\left[I_{p p}^{r}\right]_{i j}+\left[I_{p p}^{s}\right]_{i j}$ and finally

$$
\left[I_{p W}\right]_{i j}=I_{p W}\left(r_{i}, r_{j}\right)=\rho_{\infty}[I]
$$

Combining the system of equations (2.17), (2.23) and (2.29), is obtained the equation that relates the pressure over the membrane $\{P\}$ with the acceleration $\{\ddot{W}\}$

$$
\{P\}=\left[\Lambda_{p w}\right]\{\ddot{w}\}
$$

where the matrix $\left[\Lambda_{p W}\right]$ is obtained resolving the system(2.17), (2.23) and (2.29)

In a similar way it is easy to obtain the equation that relates the pressure $\left\{P_{a}\right\}$ and $\left\{P_{h}\right\}$ over the fixed boundaries with the membrane acceleration $\{\ddot{W}\}$

$$
\left\{P_{h}\right\}=\left[\Lambda_{h W}\right]\{\ddot{W}\}
$$




$$
\left\{P_{a}\right\}=\left[\Lambda_{a w}\right]\{\ddot{W}\}
$$

\section{Calculus of the membrane frequencies}

Taking into account that $\ddot{W}=-\omega^{2} W$ and defining $\left[\Lambda_{p w}\right]=\rho_{\infty} a\left[\bar{\Lambda}_{p W}\right]$, the pressure over the membrane is obtained as $\{P\}=-\rho_{\infty} a \omega^{2}\left[\bar{\Lambda}_{p W}\right]\{W\}$

The next step is to solve equation (2.1). After the substitution of equations (2.4), (2.5) and (2.9), this equation is expressed as:

$$
T \Delta W_{m}^{n}(r)+\rho_{m} t_{h} \omega^{2} W_{m}^{n}(r)=P_{m}^{n}(r)
$$

By multiplying equation (2.38) by the mode $W_{u}^{v}$ and the integration over the surface of the membrane, the following system is obtained:

$$
\iint_{S_{m}} T \Delta W_{m}^{n} W_{u}^{v} q_{m}^{n} d S+\rho_{m} t_{h} \omega^{2} \iint_{S_{m}} W_{m}^{n} W_{u}^{v} q_{m}^{n} d S=\iint_{S_{m}} P_{m}^{n} W_{u}^{v} d S
$$

After applying a collocation method and considering the expression (2.37) for the pressure over the membrane , the following scalar equation is obtained:

$$
\begin{aligned}
& T\left\lfloor\Delta W_{m}^{n} \mid[\Delta S]\left\{W_{u}^{v}\right\} q_{m}^{n}+\rho_{m} t_{h} \omega^{2}\left\lfloor W_{m}^{n}\right\rfloor[\Delta S]\left\{W_{u}^{v}\right\} q_{m}^{n}=\right. \\
& =-\rho_{\infty} a \omega^{2}\left\lfloor W_{m}^{n}\right\rfloor\left[\left[\bar{\Lambda}_{p W}\right]^{-1}\right]^{T}[\Delta S]\left\{W_{u}^{v}\right\} q_{m}^{n}
\end{aligned}
$$

Making a variation of $m, n, u, v=0,1,2, \ldots \ldots \ldots . ., N$ the following system is obtained leading to an eigenvalue problem:

$$
\left[[K]-\omega^{2}\left([M]+\left[M_{F}\right]\right)\right]\{q\}=\{0\}
$$

where the elements of the three matrices are calculated:

$$
\begin{gathered}
K_{m u}^{n v}=T\left\lfloor\Delta W_{m}^{n} \mid[\Delta S]\left\{W_{u}^{v}\right\}\right. \\
M_{m u}^{n v}=-\rho_{m} t_{h}\left|W_{m}^{n}\right|[\Delta S]\left\{W_{u}^{v}\right\} \\
\left.M_{F m u}^{n v}=-\rho_{\infty} a \mid W_{m}^{n}\right\rfloor\left[\left[\bar{\Lambda}_{p W}\right]^{-1}\right]^{T}[\Delta S]\left\{W_{u}^{v}\right\}
\end{gathered}
$$

It should be noted that the matrix $\left[M_{F}\right]$ for compressible cases is a complex matrix and will depend on the natural frequencies through the wave number $k=\frac{\omega}{a_{\infty}}$. Thus, the solution to equation (2.41) for the determination of the natural frequencies $\omega$ becomes a nonlinear eigenvalue problem since the fluid-mass matrix $\left[M_{F}(\omega)\right]$ depends on the frequency.

An iteration procedure needs to be used to obtain the natural frequencies of the system. The iteration scheme is developed as follows:

For each frequency the above procedure converges into two or three iterations. It should be noted that for a compressible fluid the natural frequencies are obtained one by one, while for an incompressible fluid all of them are obtained at the same time. Once the natural frequencies of the coupled fluid-structure are determined, the normal modes can be computed by determining the eigenvector $\left\{q_{i}\right\}$ and expressed as a linear combination of the normal modes of the structure in vacuum.

\section{Results}


For verification of the method, in Table 1, the frequencies of a plate in contact with fluid and in vacuum $f_{l m}^{n}$ and $f_{v m}^{n}$ are compared with the results of Tariverdilo et al. [2013] for the case of a cylindrical container filled with water (incompressible fluid) with a bottom vibrating plate. For this case , the entire procedure is similar to that of the membrane and the equation (2.1) is substituted by the equation of motion of the plate, from Wang [2014]:

$$
D \nabla^{4} w(r, \theta, t)+\rho_{p} t \frac{\partial^{2} w}{\partial t^{2}}=p(r, \theta, t)
$$

with $D=\frac{E \cdot t_{h}^{3}}{12\left(1-v^{2}\right)}$ the flexural rigidity, $E$ the elasticity modulus of the material, $t_{h}$ the plate thickness, $v$ the Poisson modulus and $\rho_{m}$ the material density of the plate.

Moreover, in this case the functions $W_{m}^{n}$ are the modes of deformation of the plate that have the general expression, see Wang [2014]: $W_{m}^{n}(r)=J_{m}\left(\beta_{m}^{n} r\right)+\lambda_{m}^{n} I_{m}\left(\beta_{m}^{n} r\right)$ where $J_{m}$ and $I_{m}$ are the Bessel function and modified Bessel function of the first kind of $m^{\text {th }}$ order and $\lambda_{m}^{n}$ and $\beta_{m}^{n}$ parameters that depend of the boundary condition of the plate

The frequency $f_{l m}^{n}$ is associated with the mode $W_{m}^{n}$ of the plate in contact with water, and the frequency $f_{v m}^{n}$ corresponds to the plate in vacuum, both of which are expressed in $\mathrm{Hz}$.

As it can be observed from Table 1, there is a slight difference in results regarding the present method compared with those obtained by Tariverdilo [2013], depending on the mode considered for the different values of nodal diameters $m$ and nodal circles $n$, so the validity of this method can be concluded. There is also a significant reduction in frequency in the wet case (with water) with respect to the vacuum case and this reduction is higher with the lower value of the mode considered, , see Gascón-Pérez et al [2014].

Table 1. Natural frequencies for a clamped circular steel plate(in vacuum and water) of a cylindrical container of height $h=100 \mathrm{~mm}$, radius $a=100 \mathrm{~mm}$, and thickness $t_{h}=2 \mathrm{~mm}$

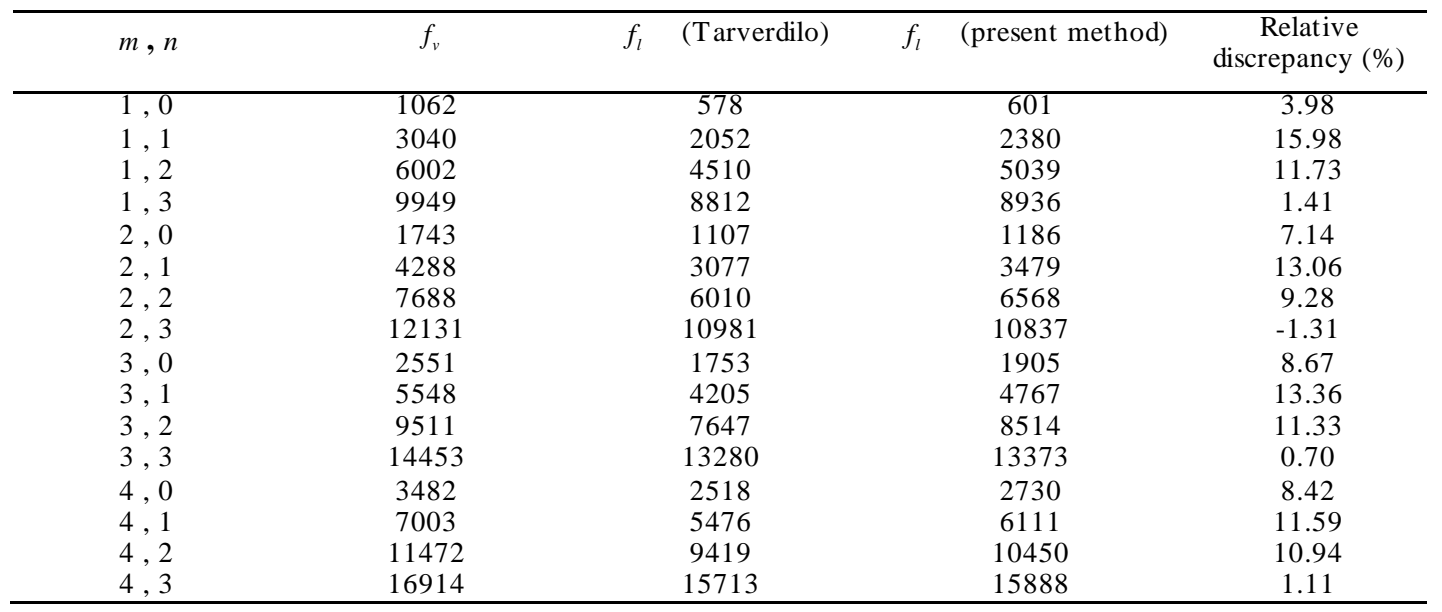

The next results are obtained by analysing the case of a drum filled with air (compressible fluid), with an aluminium bottom membrane; the following properties are considered:

Radius $a=50 \mathrm{~cm}$, thickness $t_{h}=0,02 \mathrm{~mm}$, tension $T=100 \frac{\mathrm{N}}{\mathrm{m}}$, 
material density $\rho_{m}=2700 \frac{\mathrm{kg}}{\mathrm{m}^{3}}$, drum height $h=50 \mathrm{~cm}$

Figs. 2 and 3 show the frequency variation of the membrane with the height of the drum, for different modes, with $n=0,1,2$ nodal circles and $m=0,1$ nodal diameters.

It can be seen from Fig.2 and 3, that the frequency remains constant with the height variation as it approaches to $h / a=1$, and decreases rapidly at certain threshold value of the height ratio, depending of the mode considered, indicating that the fluid mass damping effect (the fluid mass matrix) increases a lot by causing the wet natural frequency to decrease ; this value is lower as the higher mode is considered. This qualitative behaviour of frequency with height is similar to that obtained by Tariverdilo et al. [2013 ] \& [2012].

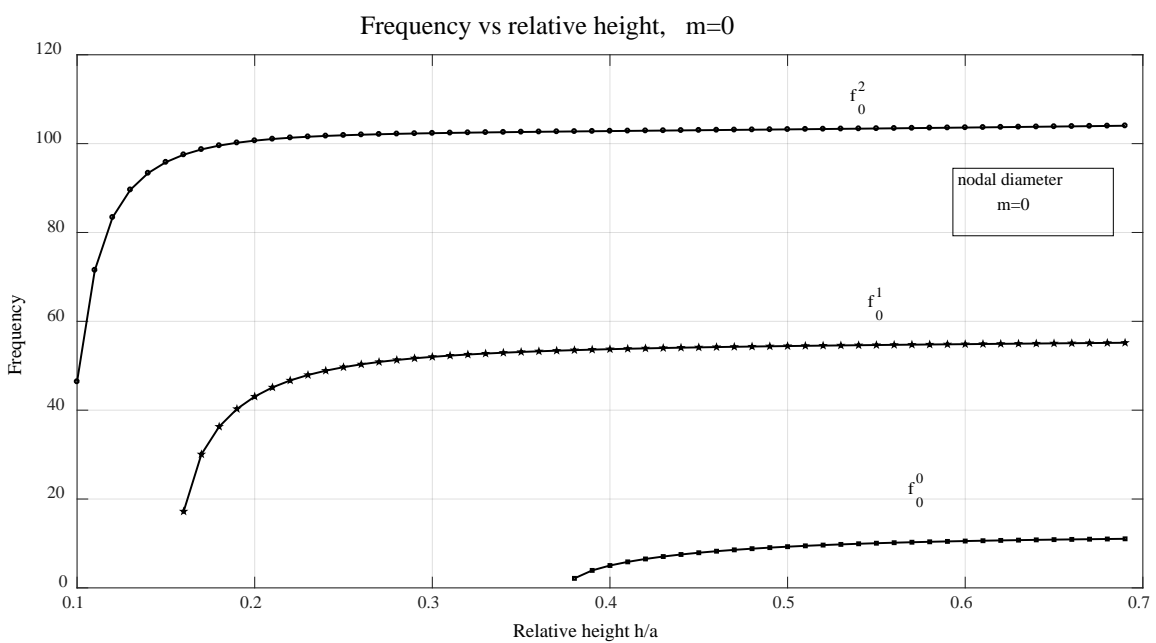

Fig. 2. Variation of membrane frequency $(\mathrm{Hz})$ with the relativeheight $h / a$ of the drum for different nodal circles $n=1,2,3$ and $m=0$ nodal diameter

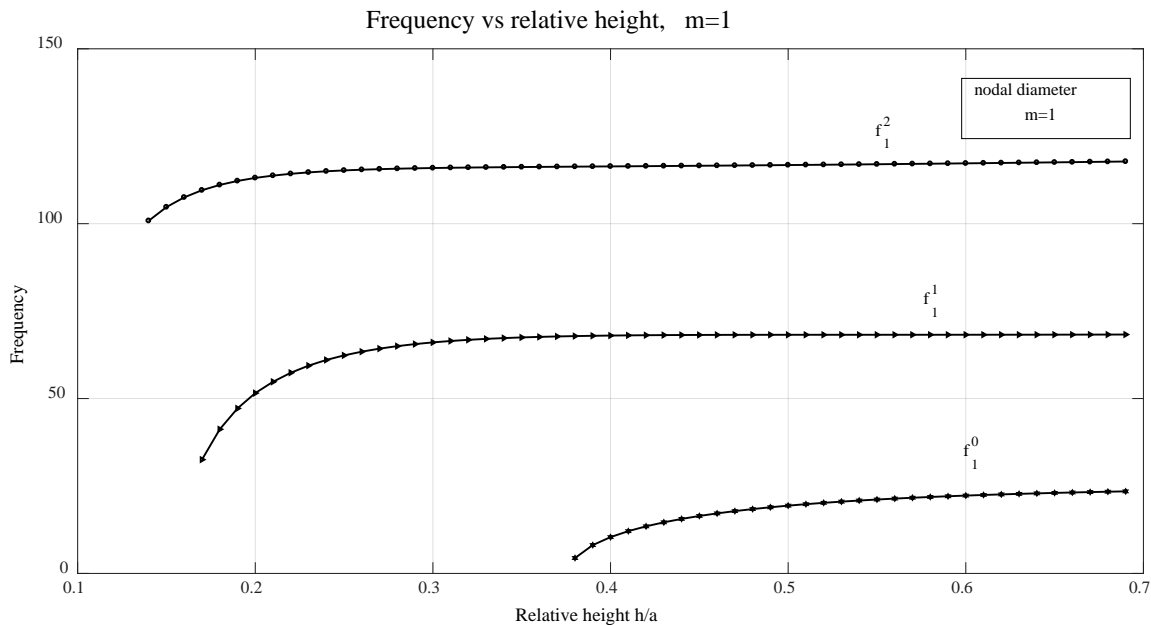

Fig. 3. Variation of membrane frequency $(\mathrm{Hz})$ with the relativeheight $h / a$ of the drum for different nodal circles $n=0,1,2$ and $m=1$ nodal diameter 
Next the variation of the frequency parameter defined as $C_{\omega}=\sqrt{\frac{\rho_{m} t_{h}}{T}} a \cdot \omega$, with different parameters of the membrane: radius $a$, thickness $t_{h}$, material density $\rho_{m}$ and tension $T$, are presented for the two cases, that is with fluid and in vacuum, and for the different modes. It must be said that in the vacuum case, the frequency parameter $C_{\omega v}$ remains constant with the variation of the respective membrane parameters.

Fig. 4 shows the variation of the frequency parameter $C_{\omega}$ with the radius $a$ of the membrane, for $m=0$ nodal diameter and $n=0,1,2$ nodal circles, where it has been taken into account that the ratio $\frac{h}{a}$ is kept constant, where $h$ is the drum height.

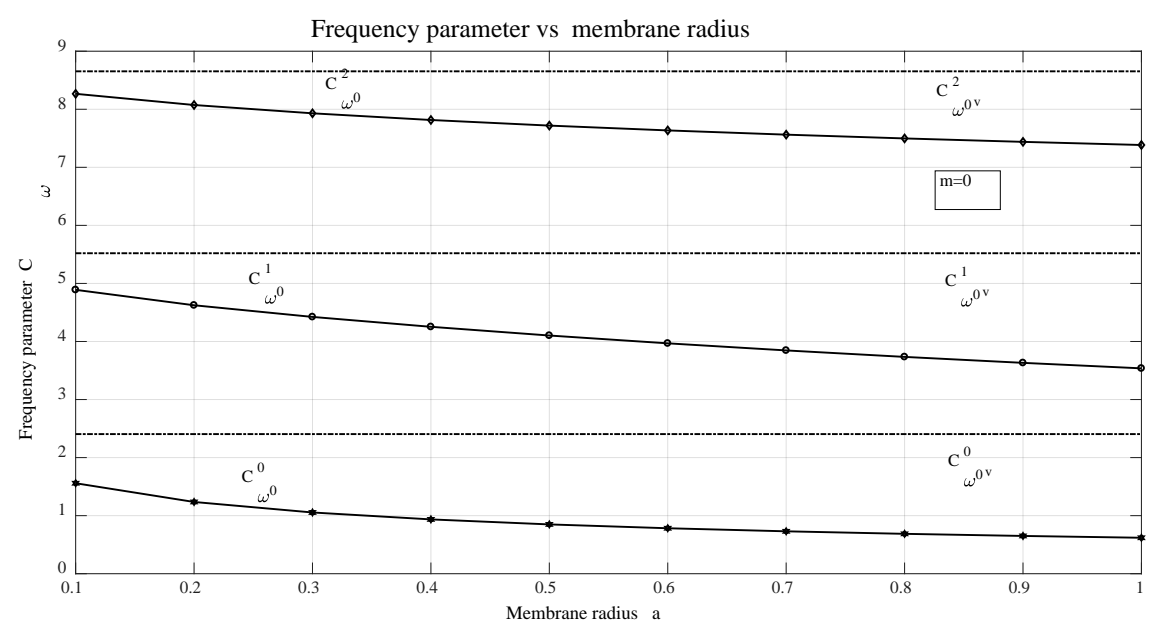

Fig. 4. Variation of membrane frequency parameter with fluid and in vacuum $C_{\omega}$ with the radius $a$ of the drum for different nodal circles $n=0,1,2$ and $m=0$ nodal diameter

This parameter decreases with the radius $a$ and the decrease rate is higher for lower values, furthermore in the same figure the frequency parameter of the membrane in vacuum(absence of fluid) appears, which is constant with the radius, while the frequency reduction effect of the fluid in respect to the vacuum case, increases with the drum size.

Fig. 5 shows the variation of the frequency parameter $C_{\omega}$ with the relative density of the fluid of the drum, for $m=0$ nodal diameter and $n=0,1$ nodal circles. The value for the reference air density is $\rho_{0}=1.225 \mathrm{~kg} / \mathrm{m}^{3}$.

This parameter decreases with the relative fluid density, and the decrease rate is higher for lower values, furthermore in the same figure the frequency parameter of the membrane in a vacuum (absence of fluid) appears, which remains constant with the relative fluid density.

Fig. 6 shows the variation of the frequency parameter $C_{\omega}$ with the membrane thickness $t_{h}$, for $m=0$ nodal diameter and $n=0,1$ nodal circles.

This parameter increases with the thickness $t_{h}$, and the increase rate is higher for lower values, furthermore in the same figure the frequency parameter of the membrane in vacuum (absence of fluid) appears, which remains constant with the thickness.

Fig. 7 shows the variation of the frequency parameter $C_{\omega}$ with the membrane material density $\rho_{m}$, for $m=0$ nodal diameter and $n=0,1$ nodal circles. 
This parameter increases with the material density $\rho_{m}$, and the increase rate is higher for lower values, furthermore in the same figure the frequency parameter of the membrane in vacuum (absence of fluid) appears, which is constant with the variation of the material density $\rho_{m}$

And finally Fig. 8 shows the variation of the frequency parameter $C_{\omega}$ with the membrane tension $T$, for $m=0$ nodal diameter and $n=0,1$ nodal circles.

This parameter remains constant with the variation of the tension $T$ in both cases, i.e. in vacuum and with fluid.

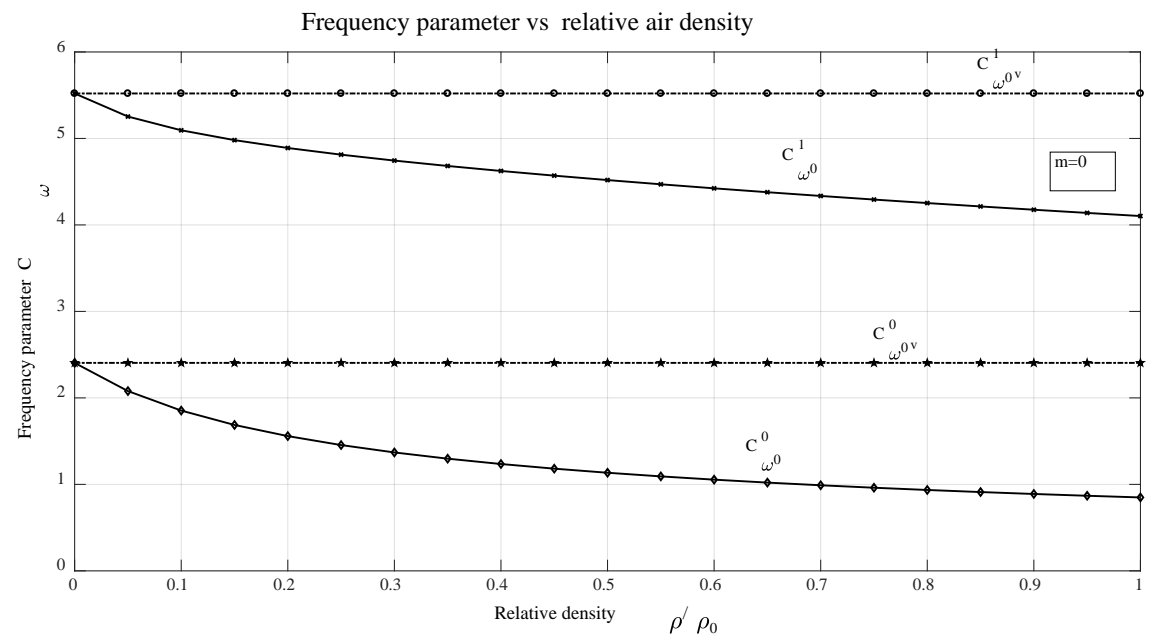

Fig. 5. Variation of membrane frequency parameter with fluid and in vacuum $C_{\omega}$ with the relative fluid density $\rho / \rho_{0}$ for different nodal circles $n=0,1$ and $m=0$ nodal diameter

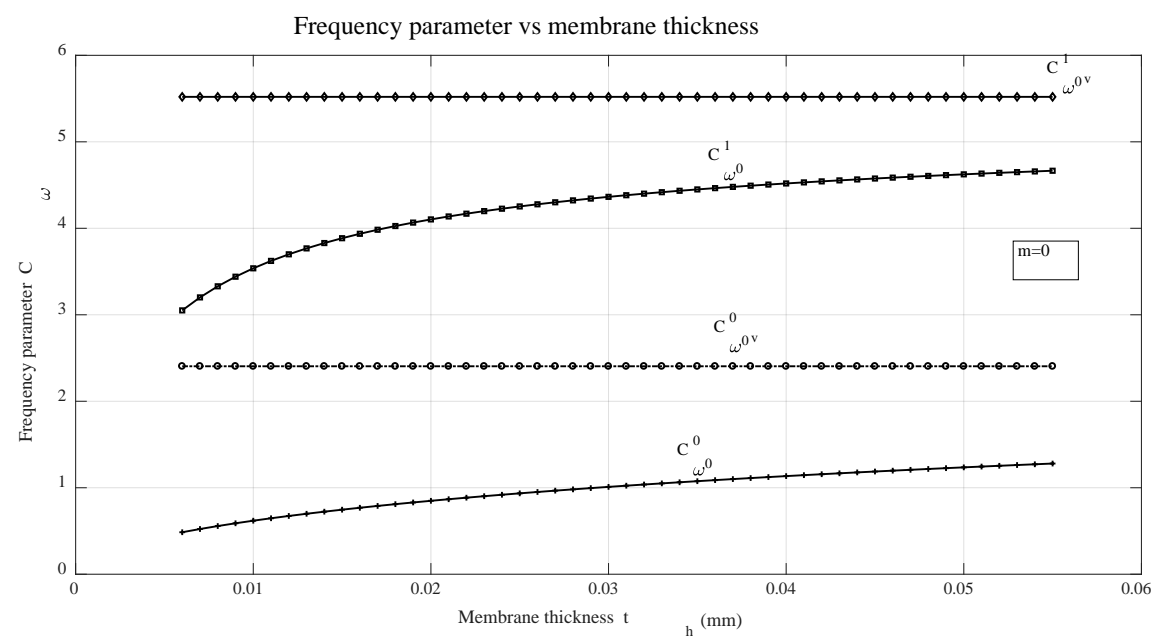

Fig. 6. Variation of membrane frequency parameter with fluid and in vacuum $C_{\omega}$ with the membrane thickness for different nodal circles $n=0,1$ and $m=0$ nodal diameter 


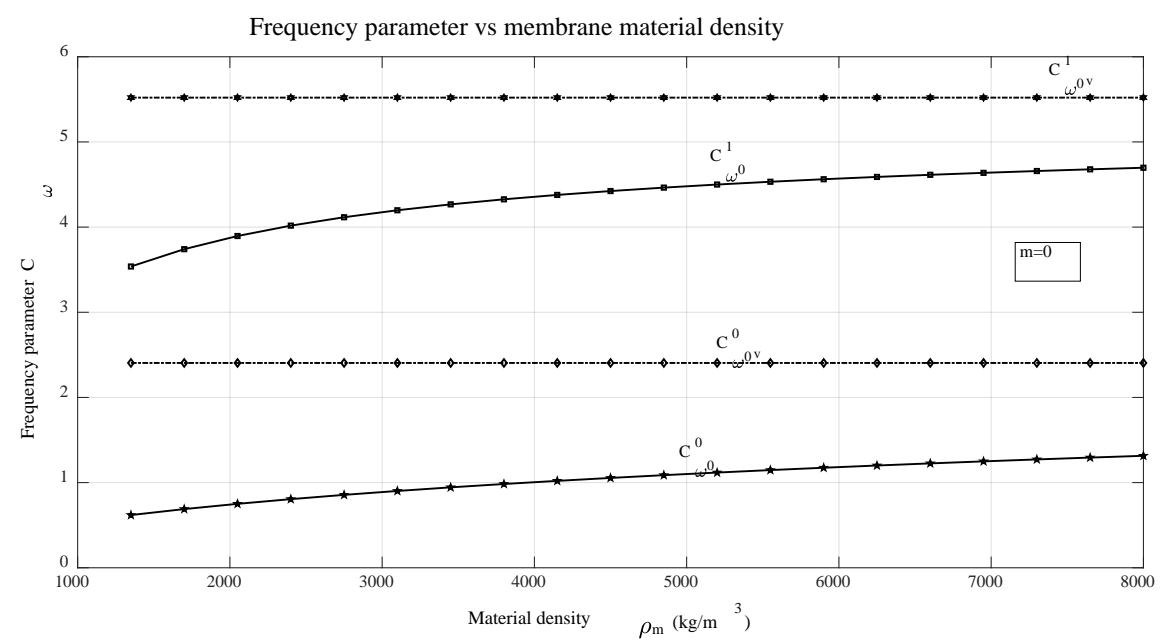

Fig. 7. Variation of membrane frequency parameter with fluid and in vacuum $C_{\omega}$ with the membrane material density for different nodal circles $n=0,1$ and $m=0$ nodal diameter

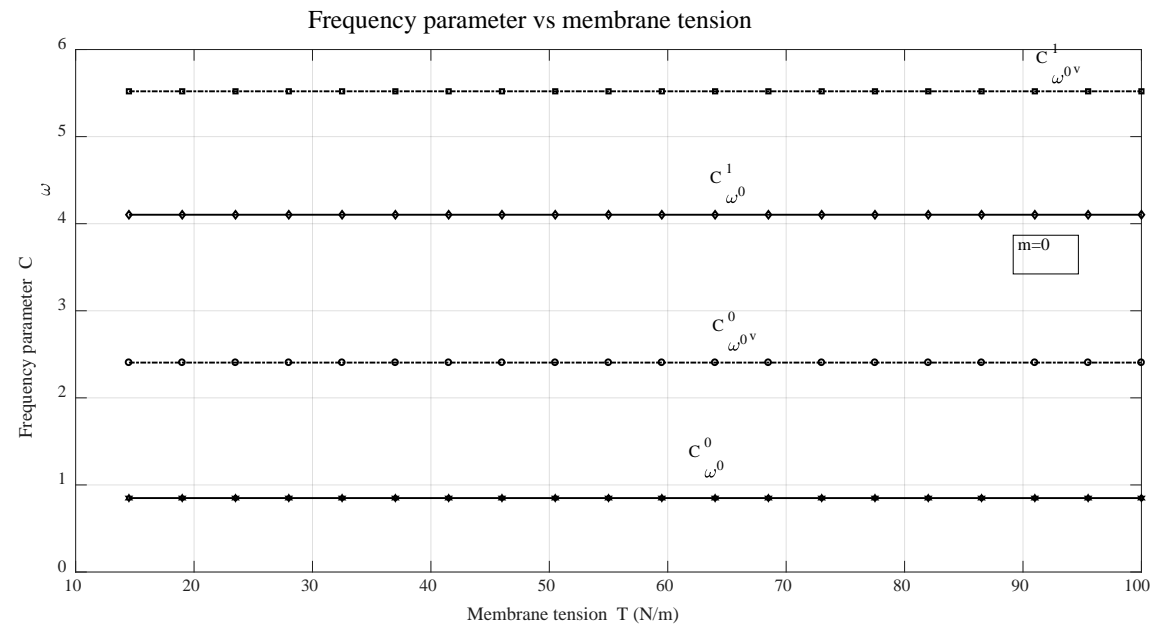

Fig. 8. Variation of membrane frequency parameter with fluid and in vacuum $C_{\omega}$ with the membrane tension for different nodal circles $n=0,1$ and $m=0$ nodal diameter

Finally, Table 2 presents the frequencies of vibration of the membrane for the different modes for a membrane drum filled with air and these are compared in respect to the values in the vacuum case. The properties of the membrane drum such as radius, thickness, tension, height and material are the considered. As it can be observed, there is a great reduction in frequency with air that is a light fluid, with respect to the vacuum case (see Fowler et al. [1987]), and this reduction is higher the lower the value of the mode.

Table 2. Natural frequencies $(\mathrm{Hz})$ for an aluminium membrane (in vacuum and air) of a cylindrical drum of height $h=50 \mathrm{~cm}$, radius $a=50 \mathrm{~cm}$, and membrane thickness $t_{h}=0.02 \mathrm{~mm}$

\begin{tabular}{cccc}
\hline$m, n$ & $f_{v}$ & $f_{a}$ & $\begin{array}{c}\text { frequency } \\
\text { reduction (\%) }\end{array}$ \\
\hline 0,0 & 32.94 & 11.68 & 64.54 \\
0,1 & 75.61 & 56.05 & 25.87
\end{tabular}




\begin{tabular}{cccc}
0,2 & 118.54 & 104.24 & 12.06 \\
1,0 & 52.49 & 24.82 & 52.71 \\
1,1 & 96.10 & 67.84 & 29.41 \\
1,2 & 139.36 & 114.34 & 17.95 \\
2,0 & 70.35 & 41.00 & 41.72 \\
2,1 & 115.30 & 87.96 & 23.71 \\
2,2 & 159.17 & 134.03 & 15.79 \\
\hline
\end{tabular}

These results are obtained in the case of air considered as compressible, but it must be said that the results obtained in the incompressible case presents a difference that is almost negligible, in particular the relative difference is in the worst case lower than $0.1 \%$. It can be said that this difference may be more appreciable in other circumstances, for example for other drum geometries or other membrane characteristics. In this regard, Fig. 9 shows the variation of the fluid mass coefficient with the wave number $k=\frac{\omega}{a_{\infty}}$. This coefficient is defined as $C_{M_{F}}=\frac{M_{F}}{\pi a^{2} h \rho_{\infty}}$ where $M_{F}$ is the diagonal term of the fluid mass matrix that is responsible for the reduction of the frequency in relation to the vacuum value, so this fluid mass coefficient can be interpreted as the transfer function that gives the effect of the fluid (pressure) over the drum membrane associated with the deformation mode of the membrane. As it can be observed the fluid mass coefficient presents an oscillating variation with the wave number, and tends to reach a value of zero for high values of the wave number $k$. It also shows its value for the incompressibility case, i.e. $k=0$.

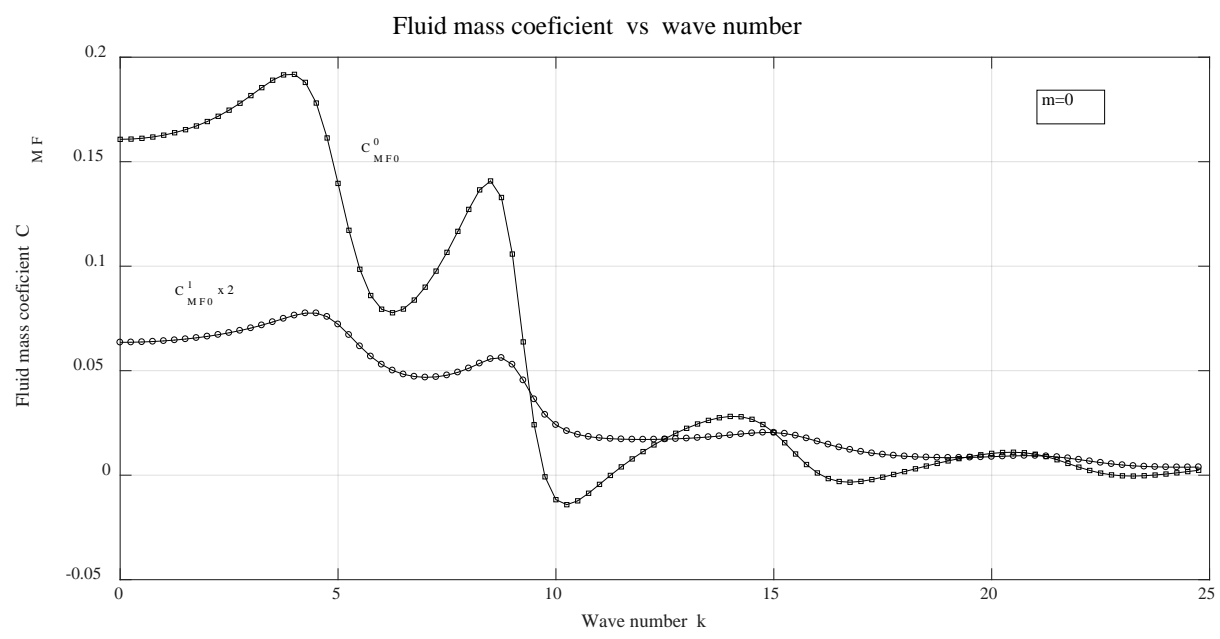

Fig.9. Fluid mass coefficient vs wave number $k$ for the membrane drum

\section{Conclusions}


The fluid-structure interaction problem has been studied for the case of a cylindrical membrane drum filled with a compressible fluid, as in the case of air. To accomplish that, a boundary element methodhas been employed and the acoustic field pressure of the fluid is calculated in order to obtain the membrane frequencies of vibration. For validation of this method, the results are compared to other methods showing a good correlation between them. The influence of different parameters of the membrane drum on his dynamic behaviour has been studied, in particular the variation of the frequency parameter with the drum height, drum radius, membrane thickness, membrane material density and fluid density. Finally, results are presented for a membrane drum filled with air and compared with respect to the vacuum case, where the compressibility effects are almost negligible; in addition the variation of the fluid mass coefficient with the wave number has been shown.

\section{References}

Abassi, W., El Baroudi, A. \& Razafimahery, F., [2016], “ Torsional vibrations of fluid-filled multilayered transversely isotropic finite circular cylinder”, Int. J. Applied Mechanics 8, (3).

Bauer,H.F., [1995], "Coupled frequencies of a liquid in a circular cylindrical container with elastic liquid surface cover”, Journal of Sound and Vibration 180, 689-704

Chiba, M., Watanabe, H. \& Bauer H.F., [2002], "Hydroelastic coupled vibrations in a cylindrical container with a membrane bottom containing liquid with surface tension”, Journal of Sound and Vibration 251, 717-740.

Crocker, M.J., [2007], Handbook of noise and vibration control, (John Wiley \& Sons, New Jersey).

Erchiqui, F., Souli, M., Kanit, T. et al, [2015], "Characterization of polymeric membranes under large deformations using fluid-structure coupling”, Int. J. Applied Mechanics 7, (5).

Fahy, F., [2001], The foundations of engineering acoustics, (Elsevier Academic Press, London).

Fowler, J., Lagerquist, N., \& Leve, H., [1987], "Effect of air in modal tests", Proceedings of the $5^{\text {th }}$ International Modal Analysis Conference, London.

Gascón-Pérez, M. \& García-Fogeda, P., [2014], "Influence of a liquid in the natural frequencies of almost circular plates", Int. J. Applied Mechanics 6, (5).

Gascón-Pérez, M. \& García-Fogeda, P, [2015], “Induced damping on vibrating circular plates submerged in still fluid ”, Int. J. Applied Mechanics 6, (5).

Jenkins, C.H.M. \& Korde, U.A., [2006], “Membrane vibration experiments: An historical review and recent results", Journal of Sound and Vibration 295, 602-613

Kyeong-Hoon, J. \& Kwi-Ja, K., [2005], “Hydroelastic vibration of a circular plate submerged in a bounded compressible fluid”, Journal of Sound and Vibration 283, 153-172

Roussos, I.M., [2014], Improper Riemann integrals, (CRC Press, Boca Raton FL).

Shabani, R., Golzar, F.G., Tariverdilo, S., Taraghi, H. et al, [2013], "Hydroelastic vibration of a circular diaphragm in the fluid chamber of a reciprocating micro pump”, International Journal of Engineering 27, 643-650

Soltani, H., \& Reddy. J.N., [2015], “ Free vibration analysis of elastic plates in contact with an inviscid fluid medium”, Int. J. Applied Mechanics 7, (3).

Tariverdilo, S., Shahmardani, M., Mirzapour, J. \& Shabani, R., [2013], “Asymmetric free vibration of circular plate in contact with incompressible fluid”, Applied Mathematical Modelling 37, 228-239.

Tariverdilo, S., Mirzapour, J., Shahmardani, M. \& Rezazadeh, G., [2012], "Free vibration of Membrane/bounded incompressible fluid”, Applied Mathematis and Mechanics 33, 1167-1178.

Wang, C.Y. \& Wang, C.M., [2014], Structural vibration: exact solutions for strings, membranes, beams and plates, (CRC Press, Boca Raton FL).

Xiang, X., Hui, Z., \& Yegao, Q., [2016], “A variational formulation for vibro-acoustic analy sis of a panel backed by an irregularly-bounded cavity”, Journal of Sound and Vibration 373, 147-653

Zhao, X, \& Li, Y., [2015], "Vibration and acoustic responses of an orthotropic composite conical shell in a hygroscopic environment”, Int. J. Applied Mechanics 7, (4). 